\title{
Computer Simulations of Regulatory Mechanisms of Hepatocyte with Hepatitis B Viruses Interconnected Dynamics
}

\author{
Mahruy Saidalieva, Mohiniso Baxromovna Hidirova*, Abrorjon Maxamatsoliyevich Turgunov \\ Scientific and Innovation Center of Information and Communication Technologies, Tashkent University of Information Technologies Named \\ After Muhammad Al-Khwarizmi, Tashkent, Uzbekistan
}

Email address:

regulatorika@yahoo.com (M. Saidalieva),mhidirova@yandex.ru (M. B. Hidirova), abrorjon26@mail.ru (A. M. Turgunov)

${ }^{*}$ Corresponding author

\section{To cite this article:}

Mahruy Saidalieva, Mohiniso Baxromovna Hidirova, Abrorjon Maxamatsoliyevich Turgunov. Computer Simulations of Regulatory Mechanisms of Hepatocyte with Hepatitis B Viruses Interconnected Dynamics. International Journal of Discrete Mathematics. Vol. 4, No. 1, 2019, pp. 32-37. doi: 10.11648/j.dmath.20190401.15

Received: February 13, 2019; Accepted: March 13, 2019; Published: April 10, 2019

\begin{abstract}
The study of the functioning of hepatitis B viruses in the liver cell using methods of mathematical modeling is considered one of the topical issues. In this article, the results on identifying of areas of regimes of the functional-differential equations of the mathematical model of regulatory mechanisms of hepatocyte with hepatitis B viruses (HBV) were presented. Characteristic modes of the regulatory of the interrelated activity of the molecular genetic mechanisms of the liver cells and viruses of hepatitis B are analyzed. The features of the area of the chaotic regime regulatory related activities of molecular genetic mechanisms of the hepatocyte and HBV by analyzing the dynamics of the Lyapunov exponent. Defined small regions with regular behavior - "r-windows" in the field of dynamic chaos. The regulatory of the hepatocyte and HBV can be moved from the region of dynamic chaos to normal region by using "r-windows". The results of the computational experiment on the quantitative analysis of the regulatory of liver cell and HBV are presented.
\end{abstract}

Keywords: Regulatory Mechanisms, Mathematical and Computer Models, Qualitative and Quantitative Analysis, Chaos, Black Hole

\section{Introduction}

Infection with HBV remains a global public health problem, so a chronic infection of $\mathrm{HBV}$ can lead to liver cirrhosis and primary hepatocarcinoma. So far, the mechanisms of the onset of liver cirrhosis and liver cancer of the viral etiology and methods of their early diagnosis are completely unknown. The development of the infectious process in hepatitis B virus is determined not only by the properties of the HBV virus, but also by individual genetically determined features of the human liver and the interrelated functioning of the molecular genetic systems of HBV and hepatocyte - liver cell (LC).

Approximately 19.4 percent of deaths in recent years have been caused by infections, and this is gradually increasing. The most deadly virus on the earth was various strains of the hepatitis virus, which kills more than 1.3 million people every year. The mortality from hepatitis increased by about 22 percent compared with HIV, tuberculosis and malaria. One of the reasons for this is that people do not even know that they have a virus. According to researchers, only 5 percent of developed and developing countries are aware of their diagnosis and treatment in medical institutions $[1,2]$. Therefore, the study of regulatory mechanisms of hepatitis B in liver cells using the methods of mathematical and computer modeling is extremely important.

Moneim I. A. and Khalil H. A. studied the global behavior of HBV spread using the SEIR model with a constant vaccination rate. Infectivity during the incubation period is considered as the second mode of transmission [3].

Wiah E. N. et al. considered that the mathematical modeling of the process of interaction of the HBV with the immune system, including the effect of therapy. To describe the interaction between populations of cells and viruses in the 
body, the authors used a system of nonlinear differential equations. In modeling, a set of various parameters that satisfy different conditions was used. The authors hypothesized on the basis of the results obtained that the model can interpret a wide range of clinical manifestations of infection [4].

Elaiw A. M. et al. considered the dynamics of $\mathrm{HBV}$ on the basis of a mathematical model using a system of nonlinear ordinary differential equations. The model includes two types of drug therapy, which are used to inhibit to the formation of viruses and prevent new infections. The model can be considered as a non-linear control system with control input, it is defined as dose-dependent drug and drug effectiveness. The authors developed treatment regimens for patients with HBV infection by using a multivariate prognostic control [5].

Chen $X$. et al. presented a modified model of the hepatitis $B$ virus with an immune response and alanine aminotransferase (ALT) periods. Most models do not take into account the role of the ALT level. However, ALT plays a major role in the detecting damage to human liver cells. Typically, an increase in ALT levels is associated with the death of uninfected hepatocytes, the natural death of infected hepatocytes, and the death of infected hepatocytes caused by the immune response. When liver cells are damaged, ALT is released into the blood, which increases the level of ALT in the serum. This, the extent of liver damage can be controlled by ALT levels. Therefore, models with ALT levels can detect the activation of the immune response and liver damage [6].

Mboya K. et al. formulated and analyzed a nonlinear mathematical model for studying mathematical modeling of optimal control of HBV infection in the presence of cytotoxic cells. The proposed model describes the interaction between normal cells, HBV and cytotoxic cells. The authors also performed numerical modeling and sensitivity analysis to determine the key parameters contributing to spread of disease, and to illustrate the analytical results. Authors carried out the numerical studies of the model in order to see the effect of key parameters on the optimal control of HBV infection in the presence of a cytotoxic agent [7].

Stanca M. C. et al. investigated the defense mechanism by developing a mathematical model for the antibody response after hepatitis B virus infection, and the model was selected for these seven infected adults identified during an acute infection and determined the ability of the virus to avoid neutralization at the expense of overproduction of noninfectious subviral particles that have HBs proteins on their surface, but do not contain a nucleocapsid protein and a viral nucleic acid series. Further, the authors showed that viral clearance can be achieved for low equilibrium levels of anti-HBV antibody, as unvaccinated in individuals, when a strong cellular immune response to controling an early infection [8].

The work of Laarabi H. et al. [9] is designed to maximize the number of normal cells (cells of the underlying liver tissue). To date, several studies have been conducted for the mathematical analysis of HBV disease control with acute and chronic stages. H. Laarabi et al. are tested the study of optimal strategies for antiviral therapy of HBV infection with the growth of logistic hepatocytes without taking into account the effect of cytotoxic cells. Therefore, M. Kissa et al. proposed to expand the work [9], including cytotoxic cells and determine optimal control over the disease.

Above mentioned numerous mathematical models describe the dynamics of the hepatitis B virus in the liver cell, mainly, the cellular level. In accordance with the biological regularity of these processes, the development of an infectious disease occurs in the relationship between the genomes of the hepatitis B viruses and liver cell. Therefore, modeling the functioning of viral hepatitis B in the liver cell, special attention should be paid to the mechanisms of molecular genetic systems of the process under consideration.

Have been proposed numerous mathematical models describing the dynamics of HBV in LC, including [3-9]. In these studies, a mathematical simulation plays an important role in the understanding and quantification of biological mechanisms that govern the dynamics of HBV. Here simulation of the hepatitis B virus is mainly performed at the cellular level. In accordance with the biological laws of these processes, the development of an infectious disease occurs when the relationship between the genomes of the LC and HBV. Therefore, the modeling of the functioning of hepatitis $B$ viruses in liver cell needs special attention in order to include the mechanisms of molecular-genetic systems of the process. We have developed a new mathematical model of the dynamics of molecular-genetic systems LC and HBV.

\section{Materials and Methods}

\subsection{Model of Hepatitis B Virus in Liver Cell}

This work is devoted to research regulatory of the LC and HBV based on functional differential equations. The equations based on biological facts and laws. A new mathematical model of the hepatitis B virus in hepatocyte developed by B. N. Hidirov, can be given as follows [10-19]:

$$
\begin{aligned}
& \varepsilon_{1} \frac{d X(t)}{d t}=\frac{a X^{2}(t-1)}{1+X^{2}(t-1)+c Y^{2}(t-1)}-X(t) ; \\
& \mathcal{E}_{2} \frac{d Y(t)}{d t}=\frac{b X(t-1) Y(t-1)}{1+d X^{2}(t-1)+Y^{2}(t-1)}-Y(t) ; \\
& X(t)=\varphi_{1}(t) ; Y(t)=\varphi_{2}(t) \text { at } t \in[0,1]
\end{aligned}
$$

where $X(t), Y(t)$ - the values characterizing the activity of the molecular genetic systems of the hepatocyte and HBV; $a, b$ - constant product formation rates of molecular genetic systems of LC and HBV; $c, d$ - parameters of the degree of interrepression of molecular genetic systems of hepatocyte and hepatitis B viruses; $\varepsilon_{1}, \varepsilon_{2}$ - parameters of the regulatory of LC and $\mathrm{HBV} ; \varphi_{1}(t), \varphi_{2}(t)$ - continuous functions on $[0,1]$. All parameters are positive.

This model describes the dynamics of interrelated activity of molecular-genetic systems of LC and HBV. The righthand-side of the equation includes activating and suppressing the forces acting on a system of LC and HBV. The qualitative 
analysis of developed equations to obtain full information about the nature of the molecular and genetic processes in any possible biologically reasonable initial conditions. The system of functional differential equations (1) is a nonlinear and closed system, and their solution can be constructed by Bellman-Cook's method of sequential integration [20].

\subsection{Model Analysis}

This model describes the dynamics of the interrelated activity of hepatocyte and hepatitis $B$ viruses at the molecular-genetic level. The system of functional-differential equations (1) is a nonlinear and closed system. Therefore, we qualitatively investigate the equilibrium position of the Equation (1). To analyze the stability of the equilibrium position of Equation (1), it is necessary to look at the equilibrium position of Equation (1) around the equilibrium position. By introducing small variable changes

$$
\begin{aligned}
& X(t)=X_{0}+x(t) ; \\
& Y(t)=Y_{0}+y(t) ;
\end{aligned}
$$

and

$$
\begin{aligned}
& X(t-1)=X_{0}+x(t-1) ; \\
& Y(t-1)=Y_{0}+y(t-1),
\end{aligned}
$$

where $X_{0}$ and $Y_{0}$ is equilibrium points for $X(t)$ and $Y(t)$; $x(t)$ and $y(t)$ is small variable of equilibrium points.

We get the following equation

$$
\begin{aligned}
& \varepsilon_{1} \frac{d X(t)}{d t}=\frac{a\left(X_{0}+x(t-1)\right)^{2}}{1+\left(X_{0}+x(t-1)\right)^{2}+c\left(Y_{0}+y(t-1)\right)^{2}}-\left(X_{0}+x(t)\right) \\
& \varepsilon_{2} \frac{d Y(t)}{d t}=\frac{b\left(X_{0}+x(t-1)\right)\left(Y_{0}+y(t-1)\right)}{1+d\left(X_{0}+x(t-1)\right)^{2}+\left(Y_{0}+y(t-1)\right)^{2}}-\left(Y_{0}+y(t)\right)
\end{aligned}
$$

We separately simplify each equation of Equation (4) obtain the following equations: equations system. As a result of the above simplifications, we

$$
\begin{aligned}
& \varepsilon_{1} \frac{d X(t)}{d t}=\left(2-\frac{2}{a} X_{0}\right) x(t-1)-\frac{2 c}{a} Y_{0} y(t-1)-x(t) \\
& \varepsilon_{2} \frac{d Y(t)}{d t}=\left(\frac{Y_{0}}{X_{0}}-\frac{2 d}{b} Y_{0}\right) x(t-1)+\left(1-\frac{2}{b} \frac{Y_{0}^{2}}{X_{0}}\right) y(t-1)-y(t) .
\end{aligned}
$$

This Equation (5) is a linearized equation for small around equilibrium positions Equation (1). We construct the characteristic equation for Equation (5). Let the solution look

as follows:

$$
x=e^{\lambda t} ; \quad x^{\prime}=\lambda e^{\lambda t} ; y=e^{\lambda t} ; y^{\prime}=\lambda e^{\lambda t} \text { and }
$$

$$
\begin{aligned}
& \left(2-\frac{2}{a} X_{0}\right) e^{-\lambda}-1-\lambda \varepsilon_{1}-\frac{2 c}{a} Y_{0} e^{-\lambda}=0 ; \\
& \left(\frac{Y_{0}}{X_{0}}-\frac{2 d}{b} Y_{0}\right) e^{-\lambda}+\left(1-\frac{2}{b} \frac{Y_{0}^{2}}{X_{0}}\right) e^{-\lambda}-1-\lambda \varepsilon_{2}=0 \text {. } \\
& \left|\begin{array}{cc}
\left.2-\frac{2}{a} X_{0}\right) e^{-\lambda}-1-\lambda \varepsilon_{1} & -\frac{2 c}{a} Y_{0} e^{-\lambda} \\
\left(\left(\frac{1}{X_{0}}-\frac{2 d}{b}\right) Y_{0}\right) e^{-\lambda} & \left(1-\frac{2}{b} \frac{Y_{0}^{2}}{X_{0}}\right) e^{-\lambda}-1-\lambda \varepsilon_{2}
\end{array}\right|=0
\end{aligned}
$$

If $\frac{1}{X_{0}}=\frac{2}{b c}$, then

$$
\begin{aligned}
& \left(2-\frac{2}{a} X_{0}\right) e^{-\lambda}-1-\lambda \varepsilon_{1}=0 \\
& \left(1-\frac{2}{b} \frac{Y_{0}^{2}}{X_{0}}\right) e^{-\lambda}-1-\lambda \varepsilon_{2}=0 .
\end{aligned}
$$

First, for the analysis of Equation (1), using the conditions of the Hayes criterion, it is necessary to arrive at the transcendental equation [21]. This is an example of an equation: $(\lambda+a) e^{\lambda}+b=0$ :

$$
\left(\lambda+\frac{1}{\varepsilon_{1}}\right) e^{\lambda}+\left(\frac{2}{a} X_{0}-2\right) \frac{1}{\varepsilon_{1}}=0
$$




$$
\left(\lambda+\frac{1}{\varepsilon_{2}}\right) e^{\lambda}+\left(\frac{2}{b} \frac{Y_{0}^{2}}{X_{0}}-1\right) \frac{1}{\varepsilon_{2}}=0 .
$$

Conditions of the Hayes criterion following conditions:

$$
\begin{gathered}
a>-1 ; \\
a+b>0 ; \\
b<\xi \sin \xi-a \cos \xi .
\end{gathered}
$$

For Equation (7) and Equation (8) we apply conditions of the Hayes criterion and we get the following conditions:

$$
\begin{gathered}
\frac{1}{2} a<X_{0}<1 \frac{1}{2} a, \\
0<\frac{Y_{0}^{2}}{X_{0}}<b .
\end{gathered}
$$

Thus, if the general conditions of the Hayes criterion Equation (9) and Equation (10) are satisfied, then the equilibrium position of Equation (1) is stable. Otherwise, it is unstable. It describes that we can observe the following

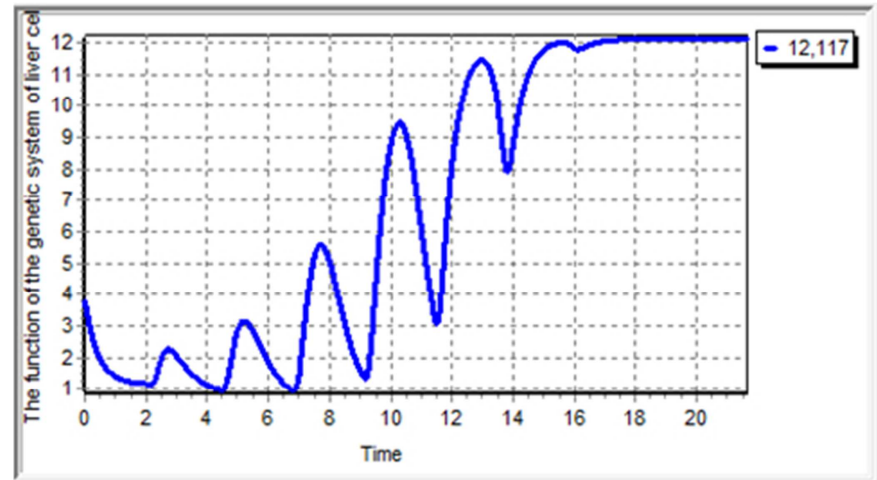

regimes of functioning of the interrelated activity of the molecular genetic systems of hepatocytes and HBV: limit cycles of the Poincare type, dynamic chaos, and the effects of the black hole.

\section{Results}

To carry out computational experiments, we created a computer model based on equations (1) using the RungeKutta method [22, 23]. Computational experiments on the quantitative analysis of the regulatory of the hepatocyte and HBV show the presence some regime (Figures 1-3).

Figure 1 shows the dominant functioning of the genetic system of the hepatocyte with the following values of the parameters

$\varepsilon_{1}=0.2 ; \varepsilon_{2}=0.04 ; a=12.2 ; b=10.6 ; c=4.1 ; d=5.8$ and $X_{0}=3.9 ; Y_{0}=6$, thus the activation of only the molecular genetic system of the hepatocyte with loss of HBV activity is described and the liver will be healthy.

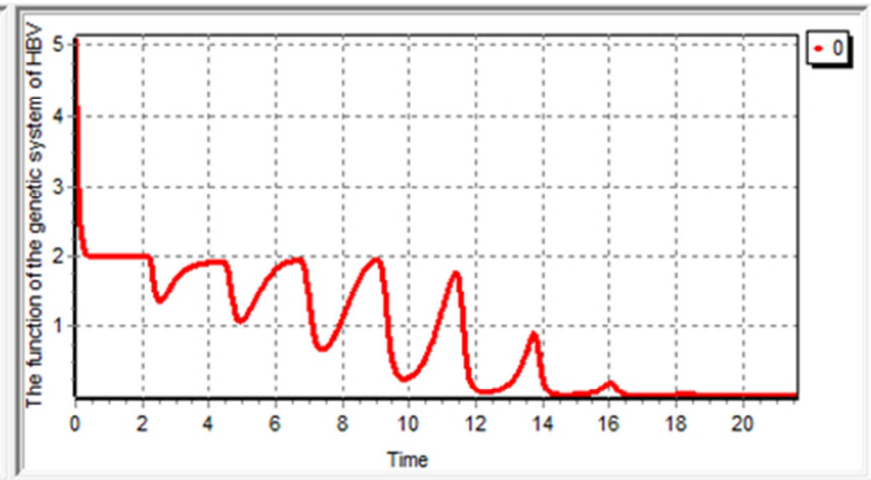

Figure 1. The regime of dominant functioning of the regulatory of hepatocyte.

Figure 2 shows the regime of the dynamic chaos regulatory of molecular genetic systems of $\mathrm{LC}$ and $\mathrm{HBV}$ with the following values of parameters $\varepsilon_{1}=0.069 ; \varepsilon_{2}=0.019 ; a=9.3 ; b=13.4 ; \quad c=4.16 ; d=7.8 \quad$ and
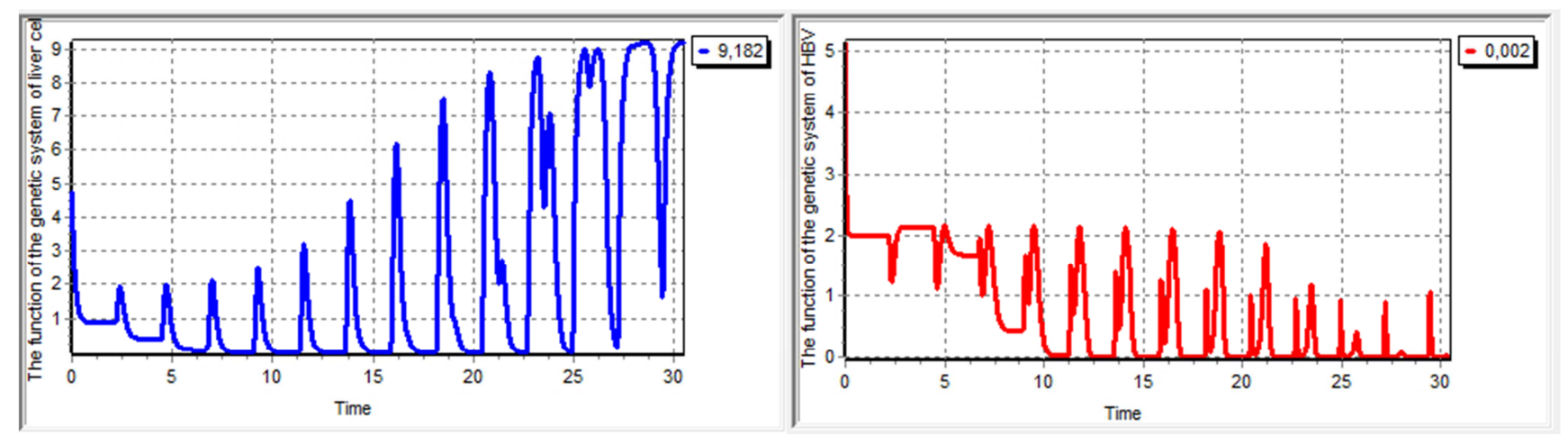

$X_{0}=5.3 ; Y_{0}=8$. The results in the Figure 2 show that the irregular functioning of the molecular genetic systems of the $\mathrm{LC}$ and HBV, and this describes the active infectious disease of viral hepatitis B in the liver.

Figure 2. The regime of the dynamic chaos of the regulatory of hepatocyte and hepatitis $B$ viruses.

In Figure 3 shows the regime of the "black hole" regulatory of molecular genetic systems of hepatocyte and hepatitis $\mathrm{B}$ viruses with the following values of parameters $\varepsilon_{1}=0.2 ; \varepsilon_{2}=0.04 ; \quad a=12.2 ; b=8.6 ; \quad c=4.1 ; d=1.8 \quad$ and $X_{0}=4.9 ; Y_{0}=4$. The obtained results in the Figure 3 show that the functioning of the molecular genetic systems of the LC 
and HBV tends to zero and destroys the body.
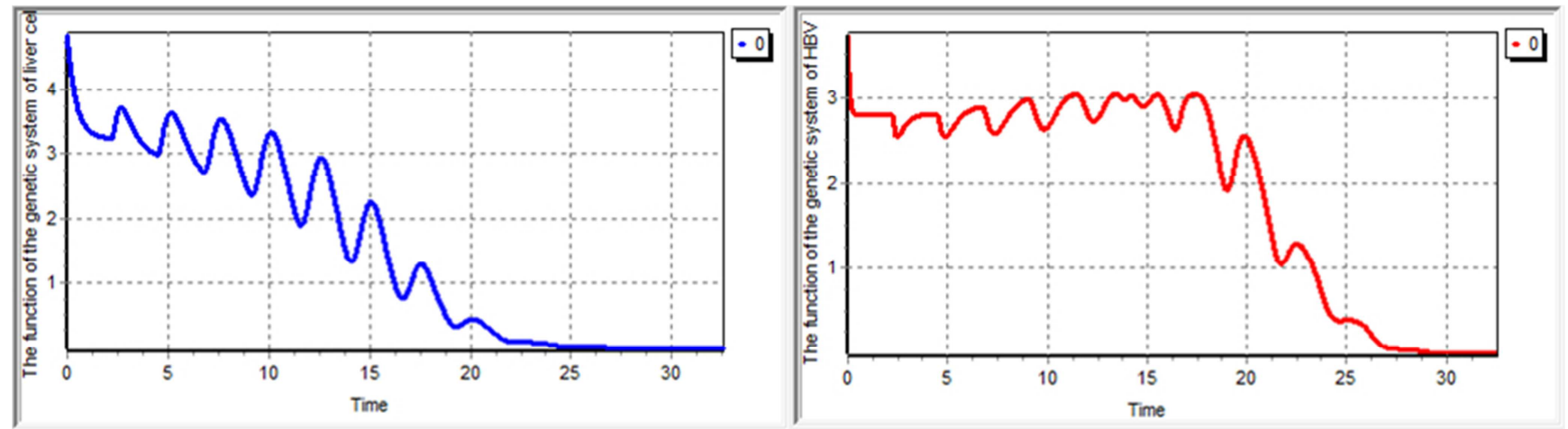

Figure 3. The regime of the "black hole" of the regulatory of hepatocyte and hepatitis $B$ viruses.

The blue graphic image shown in Figures 1-3 describes the activity of the genetic system of the hepatocyte and the red graphic image describes the activity of the genetic system of the hepatitis B viruses. The mode of irregular oscillations is characterized by a violation of the hepatocyte regulation system with a consequent deterioration of its functional activity. A quantitative study of the structural organization of the region of irregular oscillations, and the region of dynamic chaos, shows the strong inhomogeneity with sharp spasmodic changes in randomness-the Lyapunov graph. As shown by calculations on the $\mathrm{PC}$, in the regime of dynamic chaos, small regions are observed - "r-windows", within which the behavior of solutions Equation (1) has a regular character. This indicates the possibility of temporary improvement of the hepatocyte state during infection with the hepatitis

$B$ virus. However, this improvement is temporary and small perturbations are again lead molecular genetic systems of the hepatocyte into a regime of dynamic chaos. The entry into the region of irregular oscillations can be predicted: it is preceded by the series of bursts of values of the Lyapunov graph. The outbursts can be fixed by analyzing solutions on the PC. This allows predicting the onset of destructive changes in the hepatocyte under the influence of HBV. In the case of model studies of general regulatory mechanisms, the functioning of the molecular genetic system of the LC for HBV can be used for small values of $\varepsilon_{1}, \varepsilon_{2}$ the functional equation [24]

$$
\begin{aligned}
& X(t)=\frac{a X^{2}(t-1)}{1+X^{2}(t-1)+c Y^{2}(t-1)} \\
& Y(t)=\frac{b X(t-1) Y(t-1)}{1+d X^{2}(t-1)+Y^{2}(t-1)} ;
\end{aligned}
$$

and its discrete analogue

$$
\begin{aligned}
& X_{k+1}=\frac{a X_{k}^{2}}{1+X_{k}^{2}+c Y_{k}^{2}} \\
& Y_{k+1}=\frac{b X_{k} Y_{k}}{1+d X_{k}^{2}+Y_{k}^{2}} .
\end{aligned}
$$

For the analysis of chaotic regimes of the molecular genetic system of the LC in case of viral load, a computer program Lap was developed (Figure 4). $a=8.1 ; b=10.2 ; c=3.15 ; d=0.1 \quad$ and $X_{0}=1, Y_{0}=1$ are values of the parameters of the Equation (1).

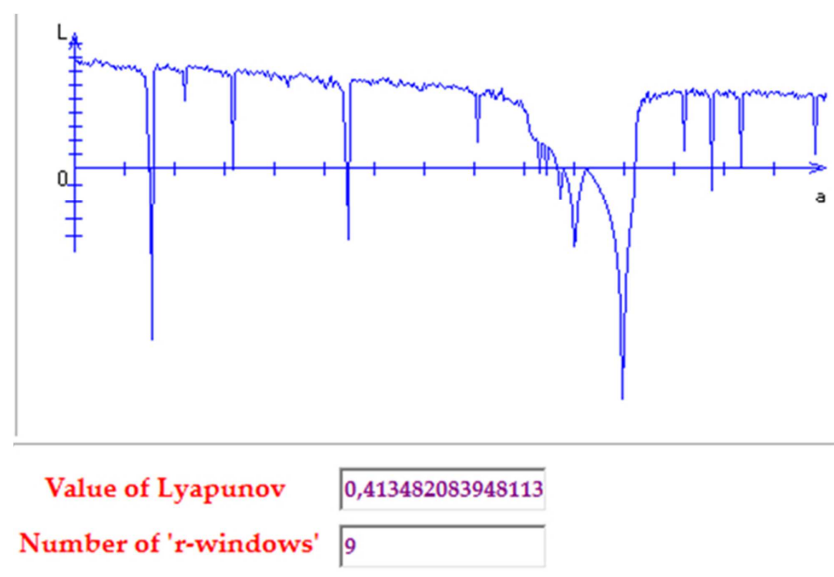

Figure 4. The Lyapunov value and Lyapunov graph for Equation (1) [25].

In Figure 4, the Lyapunov graph is located in the upper part of the axis a, that one can see the functioning of the interrelated activity of the molecular genetic systems of LC and $\mathrm{HBV}$ in the regime of dynamic chaos. But at the same time, some parts of the graph intersects the a axis and is located in the lower part. In these places, the values of Lyapunov are negative. This means that in those places the equilibrium positions are stable. It can be seen from the Figure 4 that there are 9 such stable areas called r-windows. The molecular genetic systems of LC and HBV can be moved from the region of dynamic chaos to normal region by using "r-windows".

\section{Conclusions}

Thus, the developed mathematical and computer models for the study of the functioning of the hepatocyte and HBV regulatory mechanisms allow one to assess the state of the interrelated activity of the molecular genetic systems of the $\mathrm{LC}$ and $\mathrm{HBV}$; Establish the molecular genetic basis of pathogenesis; To assess and predict the occurrence of 
characteristic stages of the course of the disease with viral hepatitis B.

Based on the results of computational experiments, it is possible to develop a parametric portrait of the functioning of regulatory mechanisms of the interrelated activity of hepatocyte and HBV. The parametric portrait includes all the regimes of the regulatory of $\mathrm{LC}$ and $\mathrm{HBV}$. The parametric portrait allows analyzing the regimes of the interrelated activity of hepatocyte and HBV at the molecular genetic level.

\section{Acknowledgements}

The authors express deep gratitude to candidate of physical and mathematical sciences B. N. Hidirov for the formulation of tasks and assistance at all stages of the study. This work was supported by grants from the Scientific and Innovation Center of Information and Communication Technologies at the Tashkent University of Information Technologies named after Muhammad Al-Khwarizmi (F4-FA-F006, A-5-005 and BV-M-F4-002).

\section{References}

[1] World Health Organization. Fact Sheet July (2016). Available at http://www.who.int/topics/hepatitis/en/

[2] Lancet. 390 (2017). pp. 1151-1210.

[3] I. A. Moneim and H. A. Khalil (2015). Modeling and Simulation of the Spread of HBV Disease with Infectious Latent, J. Appl. Math. 6: pp. 745-753. Available at http://dx.doi.org/10.4236/am.2015.65070

[4] E. N. Wiah, I. A. Adetunde and L. Brew (2012). Mathematical Modeling of the Interaction of Hepatitis B Virus with the Immune System Including the Effect of Therapy, Int. J. Mod. Math. Sci. 1(2): pp. 53-72.

[5] A. M. Elaiw, M. A. Alghamdi and Sh. Aly (2013). Hepatitis B Virus Dynamics: Modeling, Analysis, and Optimal Treatment Scheduling, Disc. Dyn. in Nat. and Soc. 9.

[6] X. Chen, K. Sun, J. Qiu, X. Chen, Ch. Yang and A. Zhang (2014). Dynamics analysis of an amended HBV infection model with a simulation for anti-HBV infection therapy, in Proceedings of the 33rd Chinese Control Conference. Nanjing. China. pp. 2829-2834.

[7] K. Mboya, D. O. Makinde, E. S. Massawe (2015). Cytotoxic Cells and Control Strategies are Effective in Reducing the HBV Infection through a Mathematical Modelling, Int. J. Prevent. and Treat. 4(3): pp. 48-57.

[8] M. C. Stanca, M. R. Ruy and S. P. Alan (2014). Antibody Responses during Hepatitis B Viral Infection, PLoS Comput. Biol. 10(7): pp. 1-16.

[9] H. Laarabi, A. Abta, M. Rachik and J. Rouyaghroumni (2013). Optimal antiviral treatment strategies of HBV infection model with logistic hepatocytes growth, ISRN Biomath.

[10] B. N. Hidirov (2014). Selected works on mathematical modeling of the regulatory of living systems, Publishing
House, Moscow, Izhevsk.

[11] B. N. Hidirov and A. M. Turgunov (2010). Mathematical modeling of regulatory mechanisms for the development of viral hepatitis B, Sci. J. Prob. Comput. and Appl. Math. 125: pp. 153-160.

[12] B. N. Hidirov and A. M. Turgunov (2012). Modeling of molecular genetics mechanisms of control of viral hepatitis B, Uzb. J. Prob. Inf. and Ener. 2-3: pp. 13-18.

[13] M. Saidalieva, M. B. Hidirova and A. M. Turgunov (2014). Areas of homogeneous solutions of the equations of the mathematical model of the regulatory of liver in hepatitis B, Uzb. J. Prob. Inf. and Ener. 6: pp. 3-8.

[14] A. M. Turgunov (2017). Characteristic regimes of the behavior of solutions of the regulatorika equations of the "Hepatocyte-HBV" system, in Mat. XVII Int. Sci. and Meth. Conf. "Informatics: Problems, Methodology, Technologies". 2: pp. $446-450$.

[15] M. B. Hidirova and A. M. Turgunov (2015). Computer modeling of infectious disease with viral hepatitis B using information technologies, in Mat. XVII Int. Sci. and Meth. Conf. "Informatics: Problems, Methodology, Technologies", 1: pp. $478-481$.

[16] M. Saidalieva, M. B. Hidirova and A. M. Turgunov (2015). Modeling of the regulatory of the liver cell in the quasistationary state of the hepatitis B virus, TUIT BULLETIN 3(35): pp. 160-165.

[17] M. B. Hidirova, M. Saydalieva and A. M. Turgunov (2016). Analysis of the molecular and genetic mechanisms of liver cells under a load of its viruses hepatitis "B", in Sci. Art. Int. Sci. Prac. Conf. "INNOVATION-2016". pp. 268-269.

[18] A. M. Turgunov (2017). On the modeling of regulatory of the liver cell and hepatitis B viruses, Sci. J. Prob. Comput. and Appl. Math. 4(10): pp. 53-62.

[19] A. M. Turgunov (2017). Analysis of the regulatory of the liver cell and hepatitis B viruses using a computer model, in Collec. Rep. Repub. Sci. Tech. Conf. IICTIDRSE, 1: pp. 263-265.

[20] R. Bellman and K. Cooke (1963). Differential Difference Equations, Publishing House, Academic Press.

[21] J. Hale (1984). Theory of Functional Differential Equations, Publishing House, Moscow, The World.

[22] V. G. Pimenov (2008). Functional-differential equations in biology and medicine, Publishing House, Tutorial, Ekaterinburg.

[23] G. Hall and J. M. Watt (1976). Modern Numerical Methods for Ordinary Differential Equations, Publishing House, Clarendon Press, Oxford.

[24] M. B. Hidirova (2014). On the solutions of the functional differential equation of the regulatory of living systems, Bul. Moscow Univ. Math. Mech. 1: pp. 50-52.

[25] B. N. Hidirov, M. Saidalieva and M. B. Hidirova (2009). Program for qualitative analysis of functional differential equations of regulatory, The software product is inspected by the State Patent Office of the Republic of Uzbekistan on 18.12.2009. Cer. Num. DGU 01879. 